\title{
What are the Associations among Inflammation, Type 2 Diabetes Mellitus and Obesity?
}

\author{
Deovina N Jordan ${ }^{1 *}$ and James L Jordan ${ }^{2}$ \\ ${ }^{1}$ Mattel Children's Hospital, Ronald Reagan University of California Los Angeles Medical Center, USA \\ ${ }^{2}$ Murrieta, USA
}

Submission: August 01, 2017; Published: August 18, 2017

*Corresponding author: Deovina N Jordan, Mattel Children's Hospital, Ronald Reagan University of California Los Angeles Medical Center, USA, Email: djjord1@yahoo.com

\section{Introduction}

Type 2 diabetes mellitus and obesity have both been associated with inflammation and with each other [1,2]. Those associations have been established with a number of physiological processes, albeit the underlying mechanisms and progressions in certain diseases of inflammation, type 2 diabetes mellitus and obesity may not be fully understood [3]. Moreover, due to the close association that exists between type 2 diabetes mellitus and obesity, attributing specific effects to either of these diseases becomes difficult [4]. Due to the large number of adverse cellular and organ level impacts attributed to inflammation, type 2 diabetes mellitus and obesity, knowing the independent and interdependent factors of these conditions can be important in health promotion, disease prevention and disease treatment. Unfortunately, the wide range of relationships among inflammation, type 2 diabetes mellitus and obesity cannot be addressed completely in a mini-review. Hence, only some examples can be briefly included herein.

One example of inflammation, type 2 diabetes mellitus and obesity impact at the cellular level is their influence on the cells of the immune system. Mast cells are activated in type 2 diabetes mellitus. These cells release inflammatory mediators (e.g., histamine) in overabundance in the pancreas of diabetic patients leading to the microvascular and macrovascular syndromes of type 2 diabetes mellitus. Yet, the exact mechanism by which mast cells and their inflammatory by-products mediate metabolic diseases is still unknown [5]. Immunoglobulin E (IgE) antibodies mediate hypersensitivity reactions to allergens and antigens. Higher levels of IgE antibodies in the serum are associated with poor glycemic control in type 2 diabetes mellitus. IgE-mediated allergic inflammation and type 2 diabetes mellitus are linked by obesity [6]. Cells of the immune system induce both low grade inflammation and insulin resistance in obesity [1].
Another example of inflammation, type 2 diabetes mellitus and obesity impact at the cellular level is their effect on the number and phenotype of adipose tissue macrophages [7]. Still another example is their influence on the pro-inflammatory mediators in adipocytes and macrophages. As a consequence of the infiltration of adipose tissue by pro-inflammatory mediators, cardiomyocyte injury and death, along with cardiac fibrosis, may develop [2]. Similarly, an example of inflammation, type 2 diabetes mellitus and obesity impact at the organ level is their influence on the cells of the immune system. The cells produce cytokines which alter insulin signaling leading to the development of insulin resistance. Free fatty acids, which are increased in obesity, start inflammatory responses in adipose tissues, liver, muscle, and pancreas. When converted to macrophages of the highly pro-inflammatory type, these free fatty acids are also known to cause insulin resistance [8].

Diabetes/ insulin resistance has been linked to nonalcoholic fatty liver disease (NAFLD) [3]. Obesity has also been linked to the development of NAFLD [1,9]. While NAFLD may not be associated with significant increases in mortality rates, it is associated with an increased risk for developing terminal liver disease [9]. In addition to impacting the liver, both type 2 diabetes mellitus and obesity have been and are also associated with increased incidence of metabolic cardiomyopathy [2] and injury to myocytes in the skeletal muscle [4]. One complication of diabetes and/or obesity at both cellular and organ levels is increased frailty among older adults [10-12]. From a physical standpoint, the main attributor to frailty is sarcopenia which has an association with inflammation [11]. However, aging itself changes with diabetes and obesity, which also contributes to increased risk for frailty [12]. Due to the complicated nature of diabetes, obesity, inflammation, and their interactions, the 
pathophysiological mechanisms of the resultant frailty remain unclear $[10,13]$. The critical research issue that is not clearly defined in the current literature is the degree to which type 2 diabetes mellitus and obesity interact and act separately in the development of inflammatory problems. Such a distinction can be valuable in preventing and treating the inflammation and comorbidities that result from type 2 diabetes mellitus and/or obesity. The authors propose that a worthwhile study could be based on comparing inflammation and inflammatory disorders in different groups of persons. The control group shall be composed of persons with no type 2 diabetes mellitus and are not obese. The second group will comprise of persons with type 2 diabetes mellitus but are not obese. The third group shall be composed of persons with both type 2 diabetes mellitus and obesity. The fourth group will comprise of persons with no type 2 diabetes mellitus but are obese. In that way, the influence of type 2 diabetes mellitus versus obesity on inflammation and inflammatory disorders could be better studied and understood.

\section{References}

1. Asghar A, Sheikh N (2017) Role of immune cells in obesity induced low grade inflammation and insulin resistance. Cell Immunol 315: 18-26.

2. Nishida K, Otsu K (2017) Inflammation and metabolic cardiomyopathy. Cardiovasc Res 113(4): 389-398.

3. Kitade H, Chen G, Ni Y, Ota T (2017) Nonalcoholic fatty liver disease and insulin resistance: new insights and potential new treatments. Nutrients 9(4): 387.
4. Varemo L, Henriksen TI, Scheele C, Broholm C, Pedersen M, et al. (2017) Type 2 diabetes and obesity induce similar transcriptional reprogramming in human myocytes. Genome Medicine 9: 47.

5. Kempuraj D, Caraffa A, Ronconi G, Lessiani G, Conti P (2016) Are mast cellsimportant in diabetes? Pol J Pathol 67(3): 199-206.

6. Sokolova RN, Yankova RK, Adabjieva TI, Popova TA, Ivanovska MV, et al. (2017) Association between type 2 diabetes, obesity and key immunological components of Ig-E mediated inflammation. Folia Med (Plovdiv) 59(2): 159-164.

7. Appari M, Channon KM, McNeil E (2017) Metabolic regulation of adipose tissue macrophage (ATM) function in obesity and diabetes. Antioxidants and Redox Signaling, pp. 1-41.

8. Ray I, Mahata SK, De RK (2016) Obesity: An immunometabolic perspective. Front Endocrinol (Lausanne) 7: 157.

9. Metrakos P, Nilsson T (2017) Non-alcoholic fatty liver disease-a chronic disease of the $21^{\text {st }}$ century. Journal of Biomedical Research 0(0): 1-9.

10. Pérez Tasigchana RF, Leön Muñoz LM, Lopez Garcia E, Gutierrez Fisacm JL, Laclaustra M, et. al. (2017) Metabolic syndrome and insulin resistance are associated with frailty in older adults: a prospective cohort study. Age Ageing 1: 1-6.

11. Perkisas S, Vandesoude M (2016) Where frailty meets diabetes. Diabetes/Metabolism Research and Reviews 32(S1): 261-267.

12. Sacha J, Sacha M, Sobon J, Borysiuk Z, Feusette P (2017) Is it time to begin a public campaign concerning frailty and pre-frailty? A review article. Front Physio 8: 484.

13. Lana A, Valdés Bécares A, Buño A, Rodriguez Artalego F, Lopez Garcia E (2017) Serumleptin concentration is associated with incident frailty in older adults. Aging Dis 8(2): 240-249.

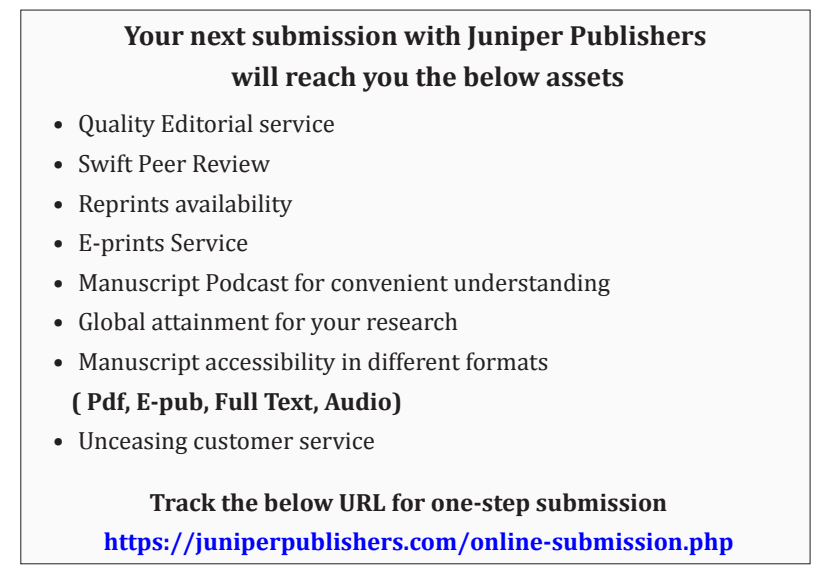

